This is an author produced version of a paper published in IEEE Journal of Selected Topics in Applied Earth Observations and Remote Sensing.

This paper has been peer-reviewed but may not include the final publisher proof-corrections or pagination.

Citation for the published paper:

Lindberg, Eva; Eysn, Lothar; Hollaus, Markus; Holmgren, Johan; and Pfeifer, Norbert. (2014) Delineation of Tree Crowns and Tree Species Classification From Full-Waveform Airborne Laser Scanning Data Using 3D Ellipsoidal Clustering. IEEE Journal of Selected Topics in Applied Earth Observations and Remote Sensing. Volume: 7, Number: 7, pp 3174-3181. http://dx.doi.org/10.1109/JSTARS.2014.2331276.

Access to the published version may require journal subscription. Published with permission from: Institute of Electrical and Electronics Engineers.

Epsilon Open Archive http://epsilon.slu.se 


\title{
Delineation of Tree Crowns and Tree Species Classification From Full- Waveform Airborne Laser Scanning Data Using 3-D Ellipsoidal Clustering
}

\author{
Eva Lindberg ${ }^{1,2}$, Lothar Eysn ${ }^{1}$, Markus Hollaus ${ }^{1}$, Johan Holmgren ${ }^{2}$, and Norbert Pfeifer ${ }^{1}$ \\ ${ }^{1}$ Department of Geodesy and Geoinformation, Vienna University of Technology \\ (TU Wien), A-1040 Vienna, Austria, \\ ${ }^{2}$ Department of Forest Resource Management, Swedish University of Agricultural Sciences, 90183 Umeå, Sweden \\ (e-mail: Eva.Lindberg@slu.se; Lothar.Eysn@geo.tuwien.ac.at; Markus.Hollaus@geo.tuwien.ac.at; Jo- \\ han.Holmgren@slu.se; Norbert.Pfeifer@geo.tuwien.ac.at) \\ Published 2014 in IEEE Journal of Selected Topics in \\ Applied Earth Observations and Remote Sensing 7(7), 3174-3181
}

\begin{abstract}
Individual tree crowns can be delineated from dense airborne laser scanning (ALS) data and their species can be classified from the spatial distribution and other variables derived from the ALS data within each tree crown. This study reports a new clustering approach to delineate tree crowns in three dimensions (3-D) based on ellipsoidal tree crown models (i.e., ellipsoidal clustering). An important feature of this approach is the aim to derive information also about the understory vegetation. The tree crowns are delineated from echoes derived from full-waveform (fwf) ALS data as well as discrete return ALS data with first and last returns. The ellipsoidal clustering led to an improvement in the identification of tree crowns. Fwf ALS data offer the possibility to derive also the echo width and the amplitude in addition to the 3-D coordinates of each echo. In this study, tree species are classified from variables describing the fwf (i.e., the mean and standard deviation of the echo amplitude, echo width, and total number of echoes per pulse) and the spatial distribution of the clusters for pine, spruce, birch, oak, alder, and other species. Supervised classification is done for 68 field plots with leave-one-out cross-validation for one field plot at a time. The total accuracy was $71 \%$ when using both fwf and spatial variables, $60 \%$ when using only spatial variables, and $53 \%$ when using discrete return data. The improvement was greatest for discriminating pine and spruce as well as pine and birch.
\end{abstract}

Keywords: Canopy layers, individual trees, light detection and ranging (lidar), single tree detection, three-dimensional (3-D) model

\section{Introduction}

Airborne laser scanning (ALS) data are measurements of reflected laser pulses from the ground and other objects, among them vegetation elements. The most common type of ALS data is discrete return data. Discrete return systems generally use a constant fraction discriminator to trigger on peaks that rise above a set threshold to record three or four peaks, often including the first and last returns, and generally include the amplitudes (i.e., intensities) of each return. Discrete return systems have a dead time between returns that is related to the length of the laser pulse. If the dead time is large, it degrades the range resolution [1]. Some ALS systems provide full-waveform (fwf) data. Fwf systems use a digitizer that samples the returned waveform (or forms, if there are multiple signals separated by periods below the threshold) and records the amplitude of the reflected laser pulse at set intervals. Fwf data can be processed with more advanced methods than the discrete return detection to derive echoes (i.e., returns) for which also the echo amplitude and the echo width can be determined [2].
Forest inventory and vegetation analyses are important applications of ALS. This study presents a new method to delineate tree crowns in three dimensions (3-D) from ALS data to derive information about trees and larger shrubs, especially below the topmost canopy layer. Additionally, information derived from fwf ALS data is used for tree species classification of each delineated tree crown. Finally, the benefit of fwf ALS data is investigated in comparison to discrete return ALS data with first and last returns for tree crown delineation and species classification.

One common way to delineate individual trees is creating a surface model, most often a canopy height model (CHM; i.e., a raster where the cell values are the heights above the ground obtained from the highest echoes within each raster cell), identifying local maxima in the surface model and delineating the areas around the local maxima with, e.g., region growing [3] or watershed segmentation [4]. The delineation can be improved by using prior knowledge about the shapes and proportions of tree crowns. For example, the proportions of the delineated segments may be restricted 
from the relative proportions of tree crowns [3]. Tree delineation based on surface models using prior knowledge has performed well in comparison with other delineation methods [5].

Delineation based on surface models identifies most of the trees in the topmost canopy layer in coniferousdominated forests. However, lower trees and shrubs below the topmost canopy layer are often not identified due to occlusion from taller trees [4, 6]. In contrast, individual trees may also be delineated from ALS data with 3-D methods, e.g., $k$-means clustering of the ALS echoes with initial positions of the cluster centers set from local maxima in a surface model $[7,8]$. Other 3-D approaches are to delineate tree crowns based on the mean shift algorithm [9] and to first determine an approximate number of tree stems clustering of the ALS data below the topmost canopy layer and then use the estimated stem number to delineate tree crowns with a normalized cut algorithm [10]. Although $k$ means clustering may include prior knowledge about the proportions of the tree crowns, 3-D methods including prior knowledge about the shape of the tree crowns have rarely been used [11].

Tree species classification of individual trees can be based on the height and amplitude distribution of the ALS data, the fit of a parabolic surface to the top of each delineated tree crown [12], or alpha shape metrics [13]. Even more accurate tree species classification can be achieved when including variables derived from fwf ALS data such as the echo width, the backscatter crosssection (i.e., the backscattering characteristics of a target depending on size, reflectivity, and the directionality of scattering), and the total number of echoes within the tree crowns [14-16]. Tree species classification has also been done for individual tree crowns delineated in 3-D based on amplitude and echo width from fwf ALS data [15].

In this study, individual tree crowns are delineated in 3-D from fwf ALS data and discrete returns based on an initial segmentation of a surface model and using prior knowledge about the shapes and proportions of the tree crowns, so-called ellipsoidal tree model clustering. A similar approach has been presented earlier [11]. However, the method in this study is an improvement of the former method and uses more information derived from the segmentation of the surface model by defining the clusters in the topmost canopy layer directly from the echoes assigned to each segment instead of using conditional distances as in the previous method. Additionally, the method in this study has fewer parameters, which makes it more transparent regarding interacting parameters and means that there are fewer parameters to calibrate for different forest types. The tree crowns are delineated both from fwf echoes (i.e., echoes derived from fwf ALS data by post-processing) and discrete returns detected by the
ALS system. Finally, the variables derived from the fwf echoes and discrete returns within each cluster are used for tree species classification.

\section{Materials}

\section{Study Area}

The study area is located in hemi-boreal forest in the southwest of Sweden (Lat. 58³0’ N, Long. 1340’ E). The main tree species are Norway spruce [Picea abies (L.)], Scots pine [Pinus sylvestris (L.)], and birch [Betula pendula (L.) and Betula pubescens (L.)]. Other tree species are oak [Quercus robur (L.)], alder [Alnus glutinosa (L.)], maple [Acer platanoides (L.)], aspen [Populus tremula (L.)], rowan [Sorbus aucuparia (L.)], and other broadleaved trees.

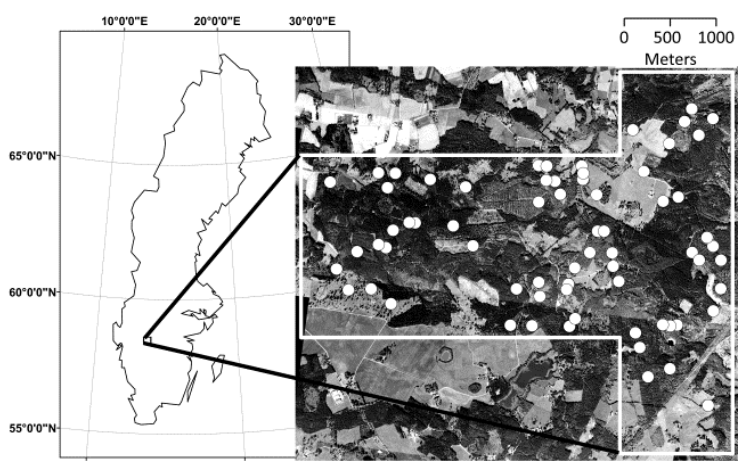

Fig. 1. Study area in Sweden (58 $30^{\circ}$ N, $13^{\circ} 40^{\prime} \mathrm{E}$ ) and positions of the field plots on a black and white aerial image.

\section{Field Data}

Data from the same field inventory have also been used in a previous study for estimation of 3-D vegetation structure from ALS data [17]. Sixty-eight circular field plots with $12 \mathrm{~m}$ radius were allocated during July and August 2009 (Fig. 1). The field inventory included only field plots where no forest management activities or other major changes of the vegetation had taken place during the last year. The field plot centers were measured using a survey grade differential GPS, which has provided a root-mean-square error of $0.27 \mathrm{~m}$ after post-processing in a separate validation.

Within the field plots, the diameter at breast height (DBH) of all trees and shrubs with $\mathrm{DBH} \geq 40 \mathrm{~mm}$ was measured using a caliper and the species was recorded. The positions of the trees and shrubs were measured relative to the plot center using an ultrasound instrument with measurement accuracy of approximately 0.3 m [18]. For a randomly selected sub-sample of trees, the height was also measured using a hypsometer. The heights of all trees were then estimated from $\mathrm{DBH}$ using a regression model (1) based on the sub-sample where tree height was measured. 
$h_{i}=\alpha_{0}+\alpha_{1} \ln \left(D B H_{i}\right)+\epsilon_{i}(1)$

where $h_{i}$ is the height of tree $i, D B H_{i}$ is the $\mathrm{DBH}$ of tree $i$, and $\varepsilon_{i}$ is the error term of the regression model. Separate models were used for spruce, pine, birch, and oak. Other deciduous tree species were included in the birch model. For the trees where the height was measured in the field, the field-measured values were used.

The mean number of trees per hectare was 736 with a standard deviation of 495 . The mean basal areaweighted height $\left(h_{B A W}\right.$; i.e., the summed product of the tree heights times the areas of the tree stems at $1.3 \mathrm{~m}$ above the ground divided by the sum of the areas) was $21.1 \mathrm{~m}$ with a standard deviation of $3.4 \mathrm{~m}$.

\section{ALS Data}

The ALS data were acquired on September 4, 2008 using a TopEye MKII ALS system with a wavelength of $1064 \mathrm{~nm}$ carried by a helicopter. The flying altitude was $250 \mathrm{~m}$ above ground, the footprint size was $25 \mathrm{~cm}$, and the measurement density was 7 emitted pulses per $\mathrm{m}^{2}$ in average. The returned fwf was measured with a sampling frequency of $2 \mathrm{GHz}$ with a maximum of 4 byte samples. The resolution of the amplitude values was 8 bits. Additionally to the fwf data, the first and last discrete returns were also stored. Laser returns were classified as ground or non-ground using a progressive triangular irregular network (TIN) densification method [19]. The ground returns were used 173 to derive a digital terrain model (DTM) with a spatial resolution of $0.5 \times 0.5 \mathrm{~m}^{2}$.

\section{Methods}

\section{Overview}

The first step of analyzing the ALS data was processing of the fwf signal to derive echoes and their attributes (Section III-B). The delineation of the tree crowns was done by segmentation of a correlation surface (CS) model for trees in the topmost canopy layer (Section III-C) followed by ellipsoidal tree model clustering of the ALS echoes in 3-D (Section III-D), both for trees corresponding to the segments from the surface model (i.e., in the topmost canopy layer) and for trees and larger shrubs below (Fig. 2). The segmentation and clustering were performed for fwf echoes as well as discrete returns detected by the ALS system.

Field-measured trees were assigned to the segments and clusters, respectively. Finally, the tree species of the delineated tree crowns were classified with supervised classification based on the spatial distribution and variables derived from the fwf of the ALS data within each tree crown (Section III-E). The success of the delineation was assessed based on the number of segments and clusters with an assigned field-measured tree within a certain distance (i.e., linked segments or clusters and trees, respectively) (Section III-F), and confusion matrices were calculated for the tree species classification.

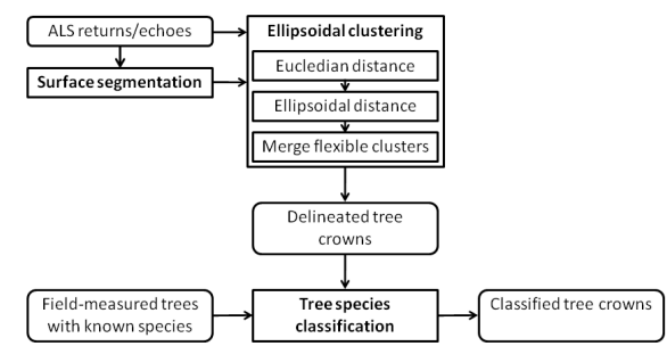

Fig. 2. Overview of the methods.

\section{Fwf Signal Processing}

This processing was done to derive individual echoes for each fwf. Each ALS fwf y was decomposed into a sum of Gaussian components $N(\mu, \sigma)$ [2] (i.e., normal distributions where $\mu$ is the mean and $\sigma$ is the standard deviation) (2)

$\hat{y}=\sum_{s=1}^{m} p_{s} N\left(\mu_{s}, \sigma_{s}\right)$

The parameter $p_{s}$ corresponds to the echo amplitude, $\mu_{s}$ corresponds to the position along the line of sight, and $\sigma_{s}$ corresponds to the echo width. The emitted pulse of the TopEye MKII system has been found to resemble a Gaussian function in an earlier study [20]. A range-corrected amplitude as was calculated by multiplying ps with the squared distance to the scanner for each echo. The decomposition was done using the expectation-maximization (EM) algorithm [21] with the method described by Lindberg et al. [17]. The maximum number of echoes per pulse was set to five to comply with the LAS specification version 1.1 [22].

\section{Segmentation of CS}

The aim of the segmentation was to establish one segment for each tree crown in the topmost canopy layer from the laser echoes (i.e., fwf echoes and discrete returns). A thorough description of the segmentation can be found in the study by Holmgren et al. [23]. The segmentation method was based on geometric tree crown models [23] and rasters with $0.25 \mathrm{~m}$ cells. For each raster cell, the height of an ellipsoid surface was calculated from different geometric tree crown models (i.e., generalized ellipsoids). Correlation coefficients were calculated between the height of the ellipsoid surfaces and the height of the ALS echoes within the horizontal model radius. The CS was defined as the 
highest correlation coefficient for each raster cell. The CS measures how well each raster cell represents a tree top. The CS was smoothed and delineated with watershed segmentation [23]. The result was delineated segments containing the tree crowns and horizontal center points corresponding to tree tops. The maximum height value $\left(H_{\text {seg }}\right)$ of the ALS echoes within each segment was defined as the segment height. The echoes were assigned to the segment within which they were located.

\section{Ellipsoidal Tree Model Clustering}

The aim of the clustering was to establish one cluster for each tree crown in the topmost canopy layer and additionally one cluster for each tree crown and larger shrub below. The algorithm was based on $k$-means clustering [24] using ellipsoidal tree crown models. Each cluster was described by its 3-D center point and comprised the laser echoes assigned to it. The idea was that the cluster center should represent the 3-D midpoint of the living crown. The clustering was done in two steps. In the first step, the laser echoes were assigned to different clusters based on the Euclidian distance between the laser echoes and the cluster centers. In the second step, an ellipsoid surface was fitted to each cluster and the laser echoes were reassigned to the different clusters based on a distance derived from the ellipsoid surface. Two categories of clusters were defined: 1) fixed clusters corresponding to tree tops already identified by segmentation of the CS; and 2) additional flexible clusters corresponding to trees below the topmost canopy layer that did not give rise to maxima in the CS. All constants were selected based on experience for the tree species and canopy conditions in the study area.

The fixed clusters were derived from the segments delineated from the CS. The horizontal coordinates of these cluster centers were set to the horizontal coordinates of the segment centers (i.e., the horizontal position of the maxima of the smoothed CS) and the vertical coordinates were initially set to $2 / 3 \times H_{\text {seg }}$ of the segments (i.e., the maximum height value of the ALS echoes within the segment), where the constant $2 / 3$ is consistent with allometric relationships for spruce, pine, and birch [25]. Additionally, for the tree crowns below the topmost canopy layer, flexible cluster centers were placed at regular horizontal distances. The total number of cluster centers was initially set to 10 times the number of delineated segments (i.e., the number of delineated segments plus nine times as many additional clusters). The regular distance was calculated to divide a circle with $40 \mathrm{~m}$ radius into quadratic grid cells corresponding to the defined number of clusters. The vertical coordinates of these cluster centers were initially set to half of the mean of $H_{\text {seg }}$ of all segments within a radius of $40 \mathrm{~m}$. The flexible cluster centers were allowed to move in 3-D, while the horizontal coordinates of the fixed cluster centers were fixed during the iterative clustering.

Initially, laser echoes were assigned to a fixed cluster if they were located inside the corresponding segment that was delineated from the surface model and at least $2.0 \mathrm{~m}$ above the DTM. For each fixed cluster, an ellipsoid surface was fitted to the topmost laser echoes $\left(x_{i}\right.$, $y_{i}, z_{i}$ ) defined as the ALS echoes with no higher ALS echoes within a horizontal distance of $0.3 \mathrm{~m}$ and within two standard deviations from the mean height above the ground of the laser echoes. The ellipsoids were used in the subsequent clustering. The center of the ellipsoid was set to the cluster center $\left(x_{j}, y_{j}, z_{j}\right)$, and the maximum height of the ellipsoid ( $h_{\text {Ellipsoid }, j}$ ) was defined as the difference between the topmost laser echo and the vertical center of the ellipsoid (3). The radius of the ellipsoid $\left(r_{\text {Ellipsoid }, j}\right)$ was selected to minimize the sum of squared residuals $\varepsilon_{i}$

$z_{j, i}=z_{j}+h_{\text {Ellipsoid }, j} \sqrt{1-\frac{\left(x_{i}-x_{j}\right)^{2}+\left(y_{i}-y_{j}\right)^{2}}{r_{\text {Ellipsoid }, j}^{2}}}+\epsilon_{i}$

where $z_{j, i}$ is the height of the ellipsoid surface $j$ (i.e., the fitted height) at the same horizontal position as laser echo $i$.

The first step of clustering was $k$-means clustering with restricted cluster center positions. For each iteration, laser echoes were assigned to the cluster center with minimum Euclidian distance $d_{\text {Euclidean }}(4)$, with the restriction that laser echoes assigned to one segment were not allowed to be assigned to a fixed cluster corresponding to a different segment

$d_{\text {Euclidean }}^{2}=\left(x_{i}-x_{j}\right)^{2}+\left(y_{i}-y_{j}\right)^{2}+\left(z_{i}-z_{j}\right)^{2}(4)$

The center of each flexible cluster center was updated to the mean of the laser echoes assigned to the cluster center if the new position was outside all ellipsoids fitted for the fixed clusters. The vertical center of each fixed cluster center was updated to the of the laser echoes if the mean was $\geq 2 / 3 \times H_{\text {seg }}$.

After completing the first step, ellipsoid surfaces were fitted to the topmost laser echoes assigned to all flexible clusters using (3) (i.e., $h_{\text {Ellipsoid }}$ and $r_{\text {Ellipsoid }}$ were estimated for each cluster). The second step of clustering used an ellipsoidal distance $d_{\text {Ellipsoid }}$ based on the ellipsoid surface fitted for each cluster. For each iteration, laser echoes were assigned to the cluster center with a minimum ellipsoidal distance $d_{\text {Ellipsoid }}$ (5)

$d_{\text {Ellipsoid }}^{2}=\frac{\left(x_{i}-x_{j}\right)^{2}+\left(y_{i}-y_{j}\right)^{2}}{r_{\text {Ellipsoid }}^{2}}+\frac{\left(z_{i}-z_{j}\right)^{2}}{h_{\text {Ellipsoid }}^{2}}(5)$

The center of each cluster center was updated with the same restrictions as in the first step, and ellipsoid surfaces were refitted to the topmost laser echoes assigned to all flexible clusters.

Finally, flexible clusters were merged pair-wise if the new cluster resulted in a smaller sum of squared resi- 
duals $\varepsilon_{i}$ of the fitted ellipsoid surface in (3). For each cluster, the candidate for merging was the closest flexible cluster and the merging started from the tallest cluster and proceeded downwards. An example of ALS data in a 2-m wide and 60-m long transect is shown in Fig. 3 together with the clusters to which the data were assigned.

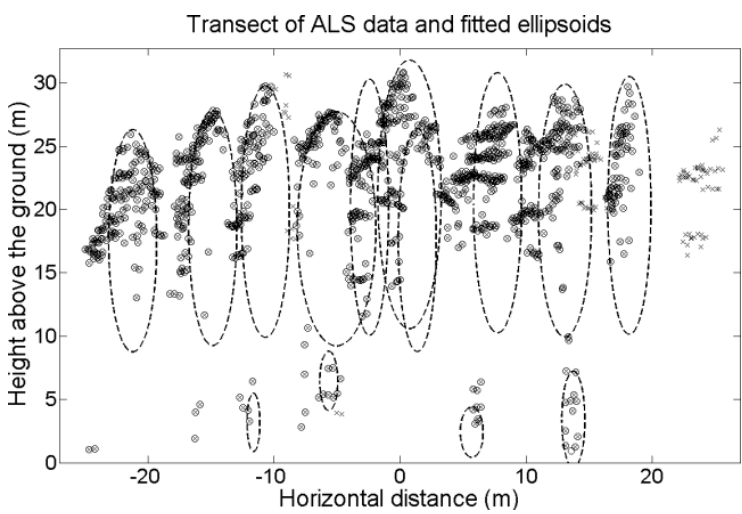

Fig. 3. An example of ALS data in a $2 \mathrm{~m}$ wide and $60 \mathrm{~m}$ long transect with data assigned to clusters inside rings. Echoes close to the ground are excluded. Clusters that are in front of each other in the viewing direction appear as overlapping. Data shown as crosses are assigned to clusters outside of the transect.

\section{Tree Species Classification}

For each cluster delineated from fwf echoes, variables (i.e., summary values for each cluster) were derived from the assigned fwf echoes to describe the spatial and fwf properties. The spatial variables were as follows.

1) The height of the ellipsoid surface ( $\left.h_{\text {Ellipsoid }}\right)$.

2) The mean normalized height (i.e., height above DTM; $\left.h_{\text {mean }}\right)$.

3) The $10^{\text {th }}, 20^{\text {th }}, \ldots$, and $90^{\text {th }}$ percentile of the normalized height $\left(h_{10}, h_{20}, \ldots\right.$, and $\left.h_{90}\right)$.

4) The total spatial variance $\left(X Y Z_{v a r}\right)$.

5) The height and radius of a parabolic surface fitted to the topmost laser echoes ( $h_{P a r}$ and $r_{P a r}$ ).

6) The ratio between the number of laser echoes assigned to the cluster relative to the total number of laser echoes within a cylinder with radius equal to the root mean square of the horizontal distance to the cluster center ( $\left.p_{\text {Ellipsoid }}\right)$.

7) The ratio between the number of laser echoes on the surface of a convex hull around the cluster relative to the total number of laser echoes assigned to the cluster $\left(p_{\text {Convex }}\right)$.

8) The number of echoes $\left(n_{t o t}\right)$.

The variables with length unit (i.e., the heights and widths) were divided by $h_{\text {Ellipsoid. }}$ The fwf variables were the mean and standard deviation of the rangecorrected amplitude of the first echoes ( $a_{\text {mean }}$ and $a_{\text {std }}$ ), echo width ( $\sigma_{\text {mean }}$ and $\sigma_{\text {std }}$ ), and number of echoes per pulse ( $m_{\text {mean }}$ and $\left.m_{\text {std }}\right)$.

The clusters linked to field-measured trees were used for tree species classification with linear discriminant analysis (LDA). The classified species were pine (P), spruce (S), birch (B), oak, alder (A), and other species (O). Two different models were used. The first model included only spatial variables and the second model included both spatial and fwf variables. A logarithmic transformation was applied for variables that were not normally distributed to fulfill the conditions of the LDA. Only variables with correlation $0.9 \%$ to each other were included in the models.

The final selections of variables were as follows.

Spatial variables only:

$h_{\text {Ellipsoid }}, h_{\text {mean }}, h_{30}, h_{60}, h_{90}, X Y Z_{\text {Var }}, h_{\text {Par }}, r_{\text {Par }}, p_{\text {Ellipsoid }}$ $p_{\text {Convex }}, n_{\text {tot }}, m_{\text {mean }}$, and $m_{\text {std }}$

Spatial and fwf variables:

$h_{\text {Ellipsoid }}, h_{\text {mean }}, h_{30}, h_{60}, h_{90}, X Y Z_{\text {Var }}, h_{\text {Par }}, r_{\text {Par }}, p_{\text {Ellipsoid }}$ $p_{\text {Convex }}, n_{\text {tot }}, a_{\text {mean }}, a_{\text {std }}, \sigma_{\text {mean }}, \sigma_{\text {std }}, m_{\text {mean }}$, and $m_{\text {std }}$

As a comparison, the tree species classification was also performed for spatial variables of the clusters delineated from discrete returns.

\section{Validation}

The field plot center positions and the orientation of the trees relative to the plot centers might contain errors. Due to this, correction was applied to the tree positions in the field data based on the segments [26].

After the correction, the clusters $i$ were linked to the field measured trees $j$ based on the distance $d_{i j}(6)$

$d_{i, j}=\sqrt{r_{x y}^{2}+\left(\frac{r_{z}}{3}\right)^{2}}$

where $r_{x y}$ is the horizontal distance and $r_{z}$ is the vertical distance between the maximum cluster height and the tree top.

The vertical distance was down-weighted due to less accurate field measurement of the tree height than the horizontal tree position. This also accounted for additional tree height errors, due to that almost one growth season separated the field inventory and the ALS data acquisition. A cluster and a field-measured tree were linked only if $d_{i j}$ was less than $1.5 \mathrm{~m}+2 \times \mathrm{DBH}$ to allow for positioning and height errors. The pairs were linked in order of increasing $d_{i j}$ and only one fieldmeasured tree or cluster was linked to each cluster or tree (i.e., one-to-one linking).

The number of linked clusters and field-measured trees was counted. Only clusters inside a field plot and at least $2 \mathrm{~m}$ from the boundary (i.e., inside a buffer zone within the field plot) were included. The procedure for the segments was similar as described for the clusters. The tree species classification was done with leave-one-out cross-validation for one field plot at a time: 1) one field plot was excluded from the dataset; 
2) the models were estimated based on the clusters and field-measured trees in the remaining field plots; and 3 ) the resulting model was used to classify the clusters in the excluded field plot.

\section{Results}

The ellipsoidal tree model clustering resulted in the greatest number of linked field-measured trees both for fwf echoes and first and last discrete returns (Table 1). However, it also resulted in a greater number of delineated tree crowns that could not be linked to any field-measured tree (i.e., commission errors). Slightly, more small field-measured trees (i.e., with a $\mathrm{DBH}<20$ $\mathrm{cm}$ ) could be linked to clusters from the ellipsoidal clustering than to segments delineated from the CS (Fig. 4). More field-measured trees could be linked to clusters and segments delineated from fwf echoes than from first and last discrete returns, but the number of clusters and segment to which no field-measured trees could linked was also greater. The total number of field-measured trees in the field plots was 2263.

TABLE 1 THE NUMBER OF LINKED TREES, CLUSTERS AND SEGMENTS; WAVEFORM ECHOES AND DISCRETE RETURNS.

\begin{tabular}{llll}
\hline \hline Method & $\begin{array}{l}\text { Linked trees } \\
\text { in field plots }\end{array}$ & $\begin{array}{l}\text { Linked } \\
\text { clusters/ } \\
\text { segments in } \\
\text { buffer zones }\end{array}$ & $\begin{array}{l}\text { Clusters/ } \\
\text { segments in } \\
\text { buffer zones }\end{array}$ \\
\hline $\begin{array}{l}\text { Ellipsoidal clustering } \\
\text { waveform echoes }\end{array}$ & $\begin{array}{l}1193 \\
(52.7 \%)\end{array}$ & $788(49.9 \%)$ & 1579 \\
$\begin{array}{l}\text { Segmentation wave- } \\
\text { form echoes }\end{array}$ & $\begin{array}{l}7143 \\
(50.5 \%)\end{array}$ & 1454 \\
$\begin{array}{l}\text { Ellipsoidal clustering } \\
\text { discrete returns }\end{array}$ & $\begin{array}{l}1121 \\
(49.5 \%)\end{array}$ & 748 (54.7\%) & 1368 \\
& & & \\
$\begin{array}{l}\text { Segmentation discrete } \\
\text { returns }\end{array}$ & $\begin{array}{l}1102 \\
(48.7 \%)\end{array}$ & & \\
\hline \hline
\end{tabular}

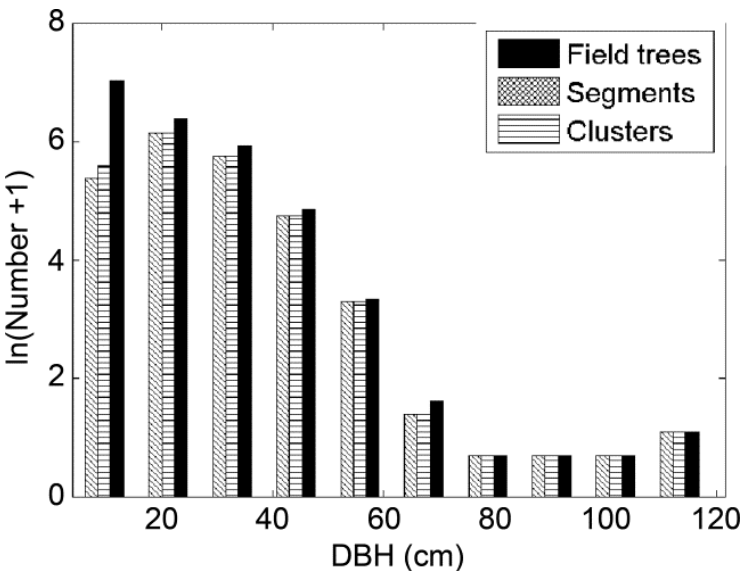

Fig. 4. The DBH distributions (log values) for waveform echoes and results from segmentation of the CS and ellipsoid clustering.

The distributions of variables derived from the fwf echoes (Section III-E) were generated for the different tree species (Fig. 5). The least overlap was found between pine and deciduous tree species, in particular for the standard deviation of the echo amplitude and the mean and standard deviation of the total number of echoes per pulse. 


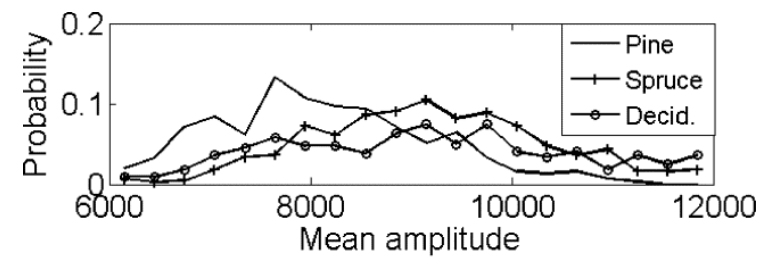

TABLE 2 CONFUSION MATRIX WHEN USING SPATIAL AND FWF VARIABLES. THE CLASSIFIED SPECIES WERE PINE (P), SPRUCE (S), BIRCH (B), OAK, ALDER (A) AND OTHER SPECIES $(O)$.
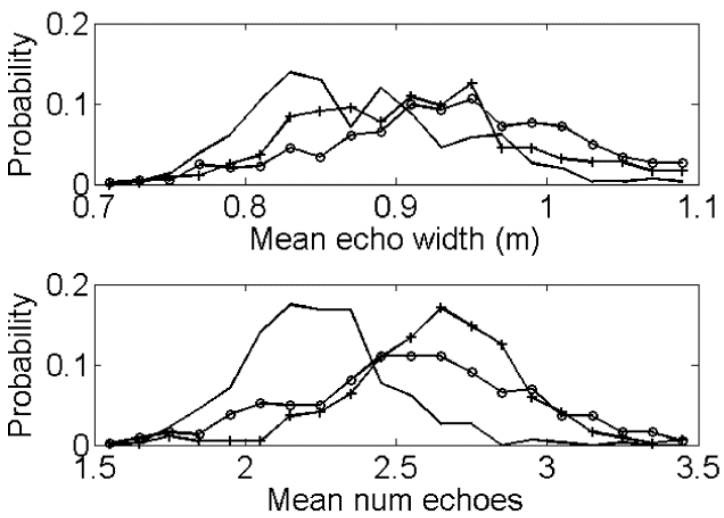

\begin{tabular}{|c|c|c|c|c|c|c|c|c|}
\hline & Field & neasu & $\mathrm{d}$ tree & pecies & & & $\dot{\Xi}$ & ن্ّ \\
\hline & P. & S. & B. & Oak & A. & O. & t0 & $\stackrel{\mathscr{n}}{\sigma}$ \\
\hline P. & 186 & 7 & 19 & 1 & 5 & 1 & 219 & 0.85 \\
\hline S. & 16 & 235 & 60 & 3 & 10 & 6 & 330 & 0.71 \\
\hline B. & 8 & 37 & 120 & 1 & 16 & 4 & 186 & 0.65 \\
\hline Oak & 0 & 2 & 4 & 0 & 0 & 4 & 10 & 0.00 \\
\hline A. & 0 & 1 & 4 & 1 & 0 & 1 & 7 & 0.00 \\
\hline O. & 0 & 6 & 5 & 7 & 2 & 17 & 37 & 0.46 \\
\hline $\begin{array}{l}\text { Tot } \\
\text { num. }\end{array}$ & 210 & 288 & 212 & 13 & 33 & 33 & 789 & \\
\hline Prod & 0.89 & 0.8 & 0.5 & 0.0 & 0.0 & 0.5 & & 0.71 \\
\hline . acc. & & 2 & 7 & 0 & 0 & 2 & & \\
\hline
\end{tabular}

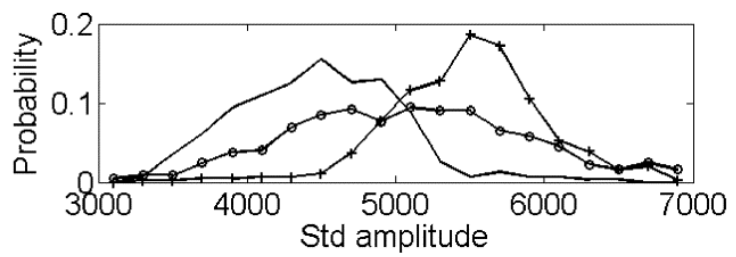

TABLE 3 CONFUSION MATRIX WHEN USING SPATIAL VARIABLES ONLY.

\begin{tabular}{|c|c|c|c|c|c|c|c|c|}
\hline & \multicolumn{6}{|c|}{ Field-measured tree species } & \multirow{2}{*}{ 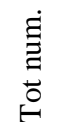 } & \multirow{2}{*}{ 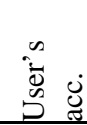 } \\
\hline & P. & S. & B. & Oak & A. & O. & & \\
\hline P. & 155 & 27 & 32 & 5 & 8 & 8 & 235 & 0.66 \\
\hline S. & 41 & 209 & 66 & 2 & 8 & 6 & 332 & 0.63 \\
\hline B. & 12 & 41 & 105 & 3 & 16 & 14 & 191 & 0.55 \\
\hline Oak & 1 & 1 & 1 & 0 & 0 & 1 & 4 & 0.00 \\
\hline A. & 0 & 0 & 3 & 0 & 0 & 0 & 3 & 0.00 \\
\hline O. & 1 & 10 & 5 & 3 & 1 & 4 & 24 & 0.17 \\
\hline $\begin{array}{l}\text { Tot } \\
\text { num. }\end{array}$ & 210 & 288 & 212 & 13 & 33 & 33 & 789 & \\
\hline $\begin{array}{l}\text { Prod. } \\
\text { acc. }\end{array}$ & 0.74 & 0.73 & 0.50 & 0.00 & 0.00 & 0.12 & & 0.60 \\
\hline
\end{tabular}

Fig. 5. Tree species differences in the probability distribution of selected fwf variables.

The tree species classification was more accurate when both fwf and spatial variables were included (Table 2; overall accuracy 0.71) than when only spatial variables were used (Table 3; overall accuracy 0.60). The improvement was greatest for discriminating pine and spruce as well as pine and birch. The biggest difference was found for pine also when using discrete return data (Table 4; overall accuracy 0.53).

TABLE 4 CONFUSION MATRIX WHEN USING DISCRETE RETURNS.

\begin{tabular}{|c|c|c|c|c|c|c|c|c|}
\hline & \multicolumn{6}{|c|}{ Field-measured tree species } & \multirow{2}{*}{$\begin{array}{l}\dot{\Xi} \\
\stackrel{\Xi}{\Xi} \\
\stackrel{0}{0} \\
\end{array}$} & \multirow{2}{*}{ 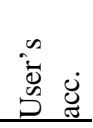 } \\
\hline & P. & S. & B. & Oak & A. & O. & & \\
\hline P. & 95 & 33 & 44 & 1 & 9 & 6 & 188 & 0.51 \\
\hline S. & 46 & 189 & 46 & 1 & 4 & 9 & 295 & 0.64 \\
\hline B. & 48 & 38 & 103 & 6 & 8 & 10 & 213 & 0.48 \\
\hline Oak & 0 & 0 & 0 & 0 & 0 & 3 & 3 & 0.00 \\
\hline A. & 6 & 2 & 7 & 0 & 5 & 4 & 24 & 0.21 \\
\hline O. & 0 & 14 & 5 & 1 & 2 & 4 & 26 & 0.15 \\
\hline $\begin{array}{l}\text { Tot } \\
\text { num. }\end{array}$ & 195 & 276 & 205 & 9 & 28 & 36 & 749 & \\
\hline $\begin{array}{l}\text { Prod. } \\
\text { acc. }\end{array}$ & 0.49 & 0.68 & 0.50 & 0.00 & 0.18 & 0.11 & & 0.53 \\
\hline
\end{tabular}




\section{Discussion}

The tree species classification was more accurate for tree crowns delineated from fwf data than those from discrete returns. More field-measured trees could be linked to clusters and segments delineated from fwf echoes than from discrete returns. The comparison is limited to discrete return ALS data with first and last returns. Some ALS systems are able to detect more than two returns [e.g., 27], which results in more measurements of the canopy. Such data might work as well as fwf data for tree crown delineation, although this study has not investigated this. However, ALS systems with first and last returns only are still common. For the fwf data, the maximum number of echoes per pulse was set to five. A test with the maximum number of echoes per pulse set to 10 gave no notable difference in the results. The fwf ALS data included in this study also made it possible to examine the echo amplitude and echo width for different tree species. The tree species showed a difference in the distributions of the amplitude, the total number of echoes per pulse, and the echo width, which is consistent with earlier studies $[14,15]$. The amplitude represents the intensity of the reflected light, whereas the echo width represents the extent of the echo along the beam. Pine showed lower mean amplitude and lower mean echo width than deciduous tree species, which may be due to that pine reflects less light because of spectral and geometrical properties and has a less extended tree crown. Additionally, pine showed a smaller number of echoes for each tree crown than the other tree species, which may be due to that pine has fewer main branches than spruce and deciduous tree species. The results suggest that tree species classification is feasible with this approach. Pine and spruce can be partly discriminated from the spatial distribution of the laser echoes [12, 13]. The variables from fwf data provided additional possibilities to discriminate pine from spruce and birch.

The ellipsoidal tree model clustering was based on the principle of using the information that can be derived about the trees in the topmost canopy layer from segmentation of a surface model and deriving additional information about lower trees and larger shrubs below the topmost canopy layer with 3-D clustering including prior knowledge about the shapes and proportions of the tree crowns. Compared to an earlier approach [11], the clustering used more information derived from the segmentation of the surface model and had fewer parameters (i.e., three parameters instead of 10) and fewer special conditions, since ellipsoids were the basis of all clusters and the distances were no longer set depending on different conditions. The ellipsoidal clustering was tested in a hemi-boreal forest. It still remains to apply the algorithm to other forest types.
More field-measured trees could be linked to clusters than to segments from the surface model. A higher measurement density or penetration rate (e.g., by using a greater scan angle) could increase the number of identified trees. However, the ellipsoidal clustering also resulted in a greater number of clusters to which no field-measured trees could be linked. Those clusters probably corresponded to parts of trees or small trees and shrubs that were not included in the field data or that had positioning errors greater than the limit which was used. Another reason for erroneous clusters might be that the ellipsoidal tree crown model was unsuitable for the laser echoes sampled from trees and shrubs below the topmost canopy layer. Ellipsoidal models have been suggested to be suitable for tree crowns, since they are intermediate shapes between cones and spheres [28] and they fulfill some common properties of tree crowns since they are symmetrical around a vertical axis and vertically elongated. Other 3-D methods also typically divide the ALS data into groups based on a distance measure and result in tree crowns with certain geometrical shapes even if it is not explicitly defined [9, 10].

The ellipsoidal models have the advantage of being straight-forward to implement with few calculations (i.e., beneficial for processing time). The clusters could be adapted to have a more peaked or egg-shaped top, but this would require additional parameters. The ellipsoidal distance used in the clustering downweighted the distance to large clusters for each laser echo, thus allowing for larger trees to have longer branches. The horizontal and vertical distances were weighted based on the proportions of the ellipsoid clusters, thus adapting the distance to the proportions of each tree crown. The surface model delineation used in this study was also based on ellipsoidal tree models and has provided good results in comparison with other methods for tree crown delineation in earlier studies [5]. This suggests that also the ellipsoidal tree model clustering performs well. The use of prior knowledge about the tree crowns has been beneficial for segmentation of surface models, and the approach in this study combines 3-D modeling and prior knowledge, which means that also trees below the topmost canopy layer can be delineated.

\section{Conclusion}

Delineation of tree crowns based on fwf ALS data identified more trees than when the delineation was based on discrete return ALS data with first and last returns. However, it also resulted in a greater number of delineated tree crowns that did not correspond to any field-measured tree. The ellipsoidal clustering identified more trees than an established surface segmentation method. For discrete return data, a greater 
number of delineated tree crowns were linked to a field-measured tree, but for fwf data a smaller number were linked.

This study is one of the few that have compared tree species classification from fwf and discrete return ALS data this far. The classification was more accurate for fwf data with the biggest improvement found for pine. The comparison is limited to discrete return ALS data with first and last returns. Additionally, tree species classification was more accurate when including variables derived from fwf than when using only variables describing the spatial distribution. The improvement was greatest for discriminating pine and spruce as well as pine and birch.

\section{Acknowledgements}

This work was supported by Karl Erik Önnesjös stiftelse för vetenskaplig forskning och utveckling and the ChangeHabitats2 project. The field inventory and the acquisition of ALS data were supported by the Hildur and Sven Wingquist Foundation.

\section{References}

[1]D. Harding, "Pulsed Laser Altimeter Ranging Techniques and Implications for Terrain Mapping," in Topographic Laser Ranging and Scanning: Principles and Processing, J. Shan and C. Toth, Eds., ed Boca Raton, FL: CRC Press/Taylor \& Francis Group, 2009.

[2] W. Wagner, et al., "Gaussian decomposition and calibration of a novel small-footprint full-waveform digitising airborne laser scanner," ISPRS Journal of Photogrammetry and Remote Sensing, vol. 60, pp. 100-112, Apr. 2006.

[3] J. Hyyppä, et al., "A segmentation-based method to retrieve stem volume estimates from 3-D tree height models produced by laser scanners," IEEE Transactions on Geoscience and Remote Sensing, vol. 39, pp. 969-975, May 2001.

[4] Å. Persson, et al., "Detecting and measuring individual trees using an airborne laser scanner," Photogrammetric Engineering and Remote Sensing, vol. 68, pp. 925-932, Sep. 2002.

[5] J. Vauhkonen, et al., "Comparative testing of single-tree detection algorithms under different types of forest," Forestry, vol. 85, pp. 27-40, Jan. 2012.

[6] S. Solberg, et al., "Single tree segmentation using airborne laser scanner data in a structurally heterogeneous spruce forest," Photogrammetric Engineering and Remote Sensing, vol. 72, pp. 1369-1378, Dec. 2006.

[7] S. Gupta, et al., "Comparative analysis of clustering-based approaches for 3-D single tree detection using airborne fullwave lidar data," Remote Sensing, vol. 2, pp. 968-989, Apr. 2010.

[8] F. Morsdorf, et al., "Clustering in Airborne Laser Scanning Raw Data for Segmentation of Single Trees," in International Archives of the Photogrammetry, Remote Sensing and Spatial Information Sciences, Dresden, Germany, 2003, pp. 27-33.
[9] A. Ferraz, et al., "3-D mapping of a multi-layered Mediterranean forest using ALS data," Remote Sensing of Environment, vol. 121, pp. 210-223, Jun 2012.

[10] J. Reitberger, et al., "3D segmentation of single trees exploiting full waveform LIDAR data," ISPRS Journal of Photogrammetry and Remote Sensing, vol. 64, pp. 561-574, Nov. 2009.

[11] E. Lindberg, et al., "Estimation of tree lists from airborne laser scanning using tree model clustering and kMSN imputation," Remote Sensing, vol. 5, pp. 19321955, Apr. 2013.

[12] J. Holmgren, et al., "Species identification of individual trees by combining high resolution LiDAR data with multi-spectral images," International Journal of Remote Sensing, vol. 29, pp. 1537-1552, 2008.

[13] J. Vauhkonen, et al., "Identification of Scandinavian commercial species of individual trees from airborne laser scanning data using alpha shape metrics," Forest Science, vol. 55, pp. 37-47, Feb. 2009.

[14] M. Hollaus, et al., "Tree species classification based on full-waveform airborne laser scanning data," in Proceedings of SilviLaser 2009, College Station, Texas, USA, 2009, pp. 54-62.

[15] J. Reitberger, et al., "Analysis of full waveform LIDAR data for the classification of deciduous and coniferous trees," International Journal of Remote Sensing, vol. 29, pp. 1407-1431, Feb. 2008.

[16] M. Rutzinger, et al., "Object-based point cloud analysis of full-waveform airborne laser scanning data for urban vegetation classification," Sensors, vol. 8, pp. 45054528, 2008.

[17] E. Lindberg, et al., "Estimation of 3D vegetation structure from waveform and discrete return airborne laser scanning data," Remote Sensing of Environment, vol. 118, pp. 151-161, Mar. 2012.

[18] T. Lämås, "The Haglöf PosTex ultrasound instrument for the positioning of objects on forest sample plots," Swedish University of Agricultural Sciences, Department of Forest Resource Management, Umeå, Sweden, Arbetsrapport 296, 2010.

[19] P. Axelsson, "DEM generation from laser scanner data using adaptive TIN models," in International Archives of the Photogrammetry, Remote Sensing and Spatial Information Sciences, Amsterdam, Netherlands, 2000, pp. 111-118.

[20] J. Reitberger, "3D-Segmentierung von Einzelbäumen und Baumartenklassifikation aus Daten flugzeuggetragener Full Waveform Laserscanner," PhD Dissertation, Institut für Photogrammetrie und Kartographie, Technische Universität München (TUM), München, 2010.

[21] A. Persson, et al., "Visualization and analysis of full-waveform airborne laser scanner data," in International Archives of the Photogrammetry, Remote Sensing and Spatial Information Sciences, Enschede, the Netherlands, 2005, pp. 103-108.

[22] ASPRS, "LAS Specification Version 1.1," ed. Bethesda, Maryland: American Society for Photogrammetry and Remote Sensing (ASPRS), 2005.

[23] J. Holmgren, et al., "Prediction of stem attributes by combining airborne laser scanning and measurements from harvesters," Silva Fennica, vol. 46, pp. 227-239, Mar. 2012. 
[24] J. MacQueen, "Some methods for classification and analysis of multivariate observations," in Proceedings of the Fifth Berkeley Symposium on Mathematical Statistics and Probability, University of California, Berkeley, California, 1967, pp. 281-297.

[25] J.-L. Widlowski, et al., "Allometric relationships of selected European tree species," Office for Official Publications of the European Communities, Ispra, Italy ISSN: 1018-5593, 2003.

[26] K. Olofsson, et al., "A method for linking fieldsurveyed and aerial-detected single trees using cross correlation of position images and the optimization of weighted tree list graphs," in Proceedings of SilviLaser
2008, 8th international conference on LiDAR applications in forest assessment and inventory, Heriot-Watt University, Edinburgh, UK, 2008, pp. 95-104.

[27] I. Korpela, et al., "Tree Species Classification Using Airborne LiDAR - Effects of Stand and Tree Parameters, Downsizing of Training Set, Intensity Normalization, and Sensor Type," Silva Fennica, vol. 44, pp. 319339, 2010.

[28] R. Nelson, "Modeling forest canopy heights: The effects of canopy shape," Remote Sensing of Environment, vol. 60, pp. 327-334, 1997. 